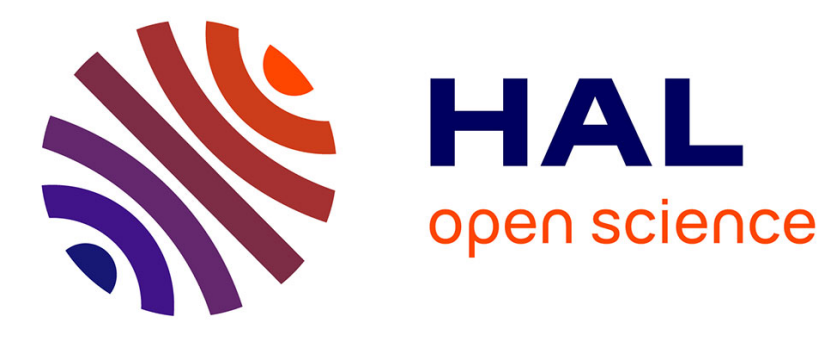

\title{
Analysis of airport ground operations based on ADS-B data
}

Michael Schultz, Xavier Olive, Judith Rosenow, Hartmut Fricke, Sameer Alam

\section{To cite this version:}

Michael Schultz, Xavier Olive, Judith Rosenow, Hartmut Fricke, Sameer Alam. Analysis of airport ground operations based on ADS-B data. 2020 International Conference on Artificial Intelligence and Data Analytics for Air Transportation (AIDA-AT), Feb 2020, Singapour, Singapore. pp.1-9, 10.1109/AIDA-AT48540.2020.9049212 . hal-03182721

\section{HAL Id: hal-03182721 \\ https://hal.science/hal-03182721}

Submitted on 26 Mar 2021

HAL is a multi-disciplinary open access archive for the deposit and dissemination of scientific research documents, whether they are published or not. The documents may come from teaching and research institutions in France or abroad, or from public or private research centers.
L'archive ouverte pluridisciplinaire $\mathbf{H A L}$, est destinée au dépôt et à la diffusion de documents scientifiques de niveau recherche, publiés ou non, émanant des établissements d'enseignement et de recherche français ou étrangers, des laboratoires publics ou privés. 


\title{
Analysis of airport ground operations based on ADS-B data
}

\author{
Michael Schultz*, Xavier Olive ${ }^{\dagger}$, Judith Rosenow*, Hartmut Fricke*, Sameer Alam ${ }^{\ddagger}$ \\ *Institute of Logistics and Aviation \\ ${ }^{\dagger}$ ONERA - DTIS \\ Technische Universität Dresden \\ Dresden, Germany \\ Université de Toulouse \\ Toulouse, France
$\ddagger$ Air Traffic Management Research Institute Nanyang Technological University Singapore

\begin{abstract}
Publicly available aircraft airborne and ground movement data pave the way to new advanced analyses of complex behaviours and collaborative decision making tools for the optimisation of airport operations. Such data-driven approaches will allow cost efficient i mplementations, $w$ hich are a key enabler for the efficient integration of s mall/medium sized airports into the air transportation network. We present an operational milestone concept based on Automatic Dependent Surveillance - Broadcast (ADS-B) messages emitted by approaching and departing aircraft. Since aircraft have to be equipped with a compliant transponder from 2020 , airports only need cheap receivers to observe operations at the runway/taxiway system and on the apron (including parking positions). These observations will allow for a systematic monitoring (using operational milestones) and predictive analytics to provide estimated values for future system states. In this contribution, we present the core elements of an innovative framework, which may bring new insights for airport operations optimisation, with a particular focus on small and medium ones. We process here aircraft movements on the ground and around Zurich airport and present four examples of applications, which will enable prediction-based decision assistance tools for efficient a irport operations.
\end{abstract}

Keywords-aircraft trajectory, operational milestones, ADS-B data, A-CDM concept, airport management

\section{INTRODUCTION}

The concept of establishing operational milestones for each aircraft movement is one core element for the airport collaborative decision making (A-CDM) [1]. These milestones should provide a common situational awareness for all stakeholders to jointly solve performance challenges at the airport, e.g. increasing punctuality during the day of operations. In this context, A-CDM is expected to improve efficiency of both airports and air transportation network, such as shortening of taxi times $(-7 \%)$, decreasing fuel burn $(-7.7 \%)$ and reducing delay caused by air traffic fl ow ma nagement $(-10.3 \%)$ [2]. In Europe, A-CDM will be implemented as part of the European Air Traffic $\mathrm{M}$ anagement $\mathrm{M}$ aster $\mathrm{P}$ lan w ithin the Single European Sky (SES) initiative [3].

Naturally, the air transportation system is both a competitive and collaborative environment, where stakeholders have to optimize their economic benefits $\mathrm{c}$ onsidering $\mathrm{v}$ arious restrictions. By giving airport stakeholders access to data from different sources, airports will be able to make more accurate predictions about their operational progress in the next period. In this context, data-driven predictions and machine learning techniques could enhance airport processes [4], since these techniques are able to show hidden interdependencies in the complex airport system. The speed and extent, with which data is shared, have massively increased over the last years and Airport Council International (ACI) Europe emphasizes the need for the digitization of aerodromes to provide a seamless transport and a resilient air transportation system [5].

Local stakeholders (ANSPs, airports, ground handlers, airlines) differ in terms of size, strategy, status, constraints, and business models. A reliable implementation have to address these distinctions appropriately, which involves various levels of collaboration and information sharing, considering the individual benefits of each stakeholder. Furthermore, imbalances between different stakeholders by means of costs and benefits have to be compensated (airlines often benefit most with small contribution efforts) [6]. A list of stakeholders and the corresponding provided information is given as follows [7]:

- Air Navigation Service Provider: estimated arrival and departure times, times based on planning, data provided by handling agent, runway in use and runway capacity

- Apron Control: landing times, in- and off- block times, start-up approval, take-off times;

- Airport Operator: stand and gate allocation, environmental information, reduction in airport capacity and in runway availability, aircraft movement data;

- Ground Handler: changes in turnaround times, target offblock time updates, planning data, possible deicing;

- Airline: flight plans, flight priorities, aircraft registrations and types.

The implementation of A-CDM is characterized by extensive negotiations between stakeholders, need for an enhanced sensor system for operational data, efficient data handling and sharing, and establishment of new procedures. At the same time, concerns are raised that shared data (information) will not only be used for A-CDM, but also for the evaluation of competitor's business structure. Beside the complex data/information sharing tasks, the estimated costs for a full A-CDM implementation is about $2.5 \mathrm{M} €$, with annual maintenance costs of $150 \mathrm{k} €$ [2], which seems only to be reasonable for major airports [8]. However, the data and information sharing in the whole air traffic network (including small/medium- 
sized airports) is a key element for a seamless and efficient transport. To significantly reduce costs for implementation and annual maintenance, we suggest to use publicly available ADS-B (automatic dependent surveillance - broadcast) data or to properly install receivers at the airport (coverage depends on available line of sight).

Furthermore, the obligation to equip aircraft with a transponder will begin in Europe in June 2020 [9], [10] and in the US almost all airspaces will be reserved only for appropriately equipped aircraft from January 2020 [11]. It is expected that current surveillance systems will be extended with ADS-B and future ground stations will be fully based on this technology, which is significantly cheaper to install and to operate. Due to the simple requirements on the receivers, ADS-B has contributed to the development of online services that display the current air traffic in real time with worldwide receiver networks (depending on the local coverage), such as The OpenSky Network [12] or Flightradar24 (flightradar24.com). This technology also offers a solution for monitoring remote areas and flights over the oceans with space-based ADS-B [13]. Furthermore, the equipment of ground vehicles at aerodromes should enable a more comprehensive monitoring of the traffic situation on the corresponding movement areas at the apron [14].

\section{A. State of the art}

In the context of aviation, several methods from the field of artificial intelligence are used to cluster [15]-[18], detect anomalies [19]-[22] and predict aircraft trajectories [23][25], develop dynamic airspace designs [26], [27], analyse runway and apron operations [21], [28]-[30], determine airport performance including the impact of local weather events [31], [32], and for airport terminal operations (turnaround) [33]. More initiatives to leverage ADS-B open data in order to improve the state of the art are already commonplace, esp. in the field of aircraft modelling [34], [35].

We consequently follow a research agenda for a datadriven management of airport operations: (a) concept of a performance-based, integrated airport management [36], (b) analysis of operational scenarios to mitigate impacts of capacity restrictions [37], (c) systematic analysis of correlations between airport performance and weather conditions at European airports [38], (d) data-driven models to forecast operational delays using neural networks [31], [32], and (e) forecast of specific parts of aircraft ground trajectory [28].

\section{B. Focus and structure of the document}

We used a data-driven airport management concept to derive operational milestones from ADS-B messages of arriving and departing aircraft [29], [30]. This approach allows to monitor the operational performance as a mandatory step to enable extended prediction capabilities, which will be focused our next research. We use Zurich airport as an example in this contribution to show that our monitoring approach could easily to be rolled out to any other airport environments without major drawbacks in the data processing.
After the introduction of the collaborative decision making in the airport environment, we provide in Section II a brief insight into the A-CDM concept. A tailored operational milestone concept (A-CDM lite) is proposed with an adapted and reduced number of milestones, all accessible from ADS-B data. In Section III we focus on the data processing in place as a proof of concept and provide an operational representation of the underlying airport operational environment. Thereafter, we show in Section IV examples of applications based on our A-CDM lite concept using a month of aircraft movement data at Zurich airport. Our contribution closes with discussion and conclusion.

\section{THE A-CDM CONCEPT}

The A-CDM concept consists of 16 milestones along the aircraft trajectory at the airport. Recommended milestones (MS) [1] are: flight plan activation, estimated off block time (look ahead time of two hours before arrival), take-off from outstation, local radar update, aircraft on final approach, landing, in-block, target start-up approval, off-block, and take off. The most important aircraft-related control parameter is the target off-block time (TOBT), which is driven by aircraft ground operations (turnaround) [39]-[41]. In particular, the progress of passenger boarding (landside operation) could mainly affect the overall operational performance [33], [42]. Thus, the aircraft take-off time is impacted by both airside and landside operations at the airport. An important connection exists between TOBT (provided by ground handling), target start-up approval time (TSAT) at the gate and target takeoff time (TTOT) at the runway (Figure 1). The TTOT also covers tactical decision of the air traffic flow and capacity management.

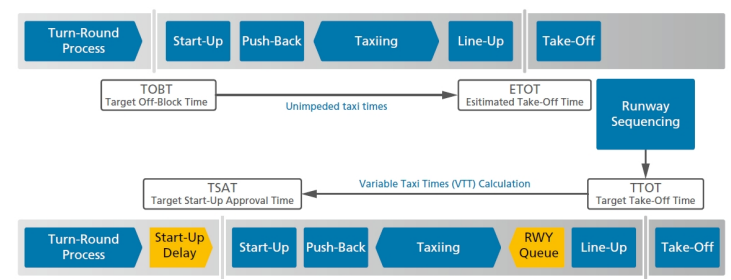

Figure 1. Calculation of Target Start-up Approval Time (TSAT) under consideration of operational milestones w.r.t. aircraft movements [43].

We extract the milestones from the aircraft status updates included in the ADS-B messages. Since these messages contain no information about operational estimated and targeted timestamps or information about ground handling operations, the initial milestone approach has to be adapted (A-CDM lite, see [29], [30]). The tailored milestone-based approach of A-CDM lite is based on the following relevant data from the ADS-B messages:

- transponder unique identifiers (to be related to tail number and aircraft type) and callsigns;

- timestamps (set by the receiver);

- positional information: latitude and longitude $\left({ }^{\circ}, 5\right.$ digit precision), altitude (ft, with steps of $25 \mathrm{ft}$ ); 

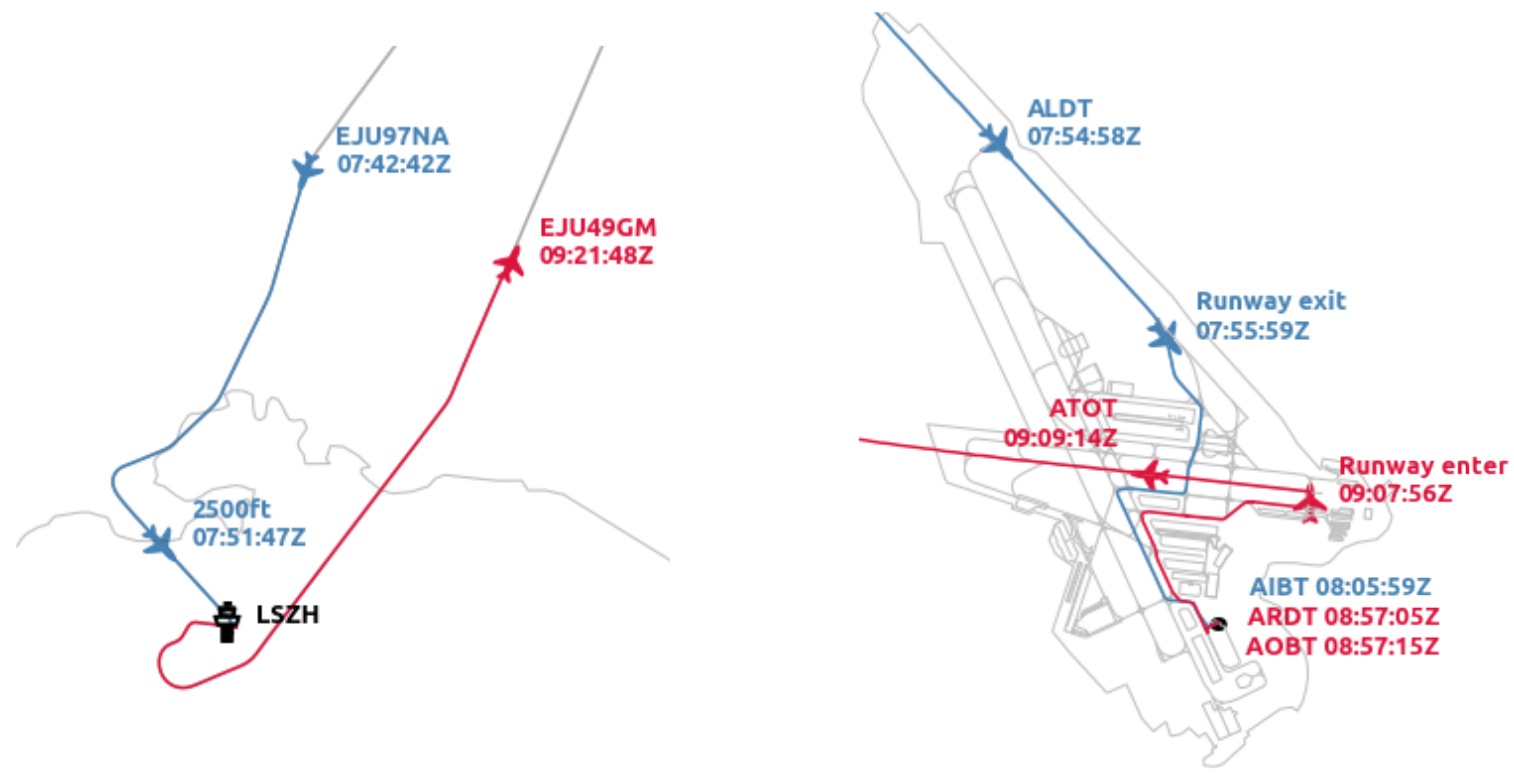

Figure 2. Tailored A-CDM milestones emphasizing airborne view (left) and airport surface view (right) of a sample aircraft movement. The arrival/ departure view shows aircraft enters the arrival sequencing and metering area (ASMA), is on final approach and leaves ASMA. On the surface, the aircraft passes the following milestones: actual landing time (ALDT), leaving runway at exit point, actual in/off-block time (AIBT, AOBT), aircraft ready time (ARDT), entering runway, actual take off time (ATOT).

- velocity information: ground speed $(\mathrm{kts})$, track angle $\left(^{\circ}\right)$ and vertical speed (ft/min).

We assign unique flight identifiers for convenience based on heuristics combining transponder identifier, callsigns and timestamps labelling collected data. In the future, we plan to use identifiers assigned to flight plan information with related in- and off-block times. In accordance with the common A-CDM milestone approach, an operational (time-based) aircraft trajectory will be described by the following numbered 10 timestamps exemplified on Figure 2: (1) aircraft entering the Arrival Sequencing and Metering Area (ASMA, the area within $40 \mathrm{~nm}$ radius around the airport, cf. [44]), (2) on final approach [29], (3) actual landing time (ALDT), (4) leaving runway at exit point, (5) actual in block time (AIBT), (6) aircraft ready time (ARDT), (7) actual off-block time (AOBT), (8) entering runway, (9) actual take-off time (ATOT), and (10) leaving ASMA.

Each milestone is extracted from ADS-B data. Milestones (1) and (10) are triggered when the aircraft enters (respectively exits) a $40 \mathrm{~nm}$ radius around the airport. Milestone (2) is set when the aircraft comes below an altitude of $2500 \mathrm{ft}$ above the airport ground [29]. Milestones (3), (4), (8) and (9) are based on geometrical constraints related to the corresponding runways. Milestone (5) is defined as the last recorded timestamp before the aircraft stops moving at the gate. Conversely, milestone (6) is set when the aircraft transponder starts to emit ADS-B messages at the gate/ apron position and (7) is set when the aircraft yields a groundspeed above a given threshold (typically $20 \mathrm{kts}$ ). Additionally, milestones (3) and (9) will be identified and verified by positional information (altitude) and velocity information (vertical speed) received from the ADS-B messages. Issues related to imprecision when dealing with message types or vertical speed references for determining the touchdown position [21] do not seem problematic for detecting take-offs.

\section{DATA PRocessing}

The framework presented in this paper heavily relies on the availability of data related to aircraft trajectories and filed flight plans. Trajectories stem from the OpenSky Network [12], a network of Mode $S$ receivers feeding data made openly available for research purposes. In order to validate the quality of the computed estimations for the milestones, a comparison has been performed based on history records of automatically filed flight plans, through Eurocontrol web services, accessed with a PREOPS license.

\section{A. $A D S-B$ data}

ADS-B is a cooperative surveillance technology, which provides situational awareness in the air traffic management system. Aircraft determine their position via satellite, inertial and radio navigation and periodically emit it (roughly one sample per second) with other relevant parameters to ground stations and other equipped aircraft. Signals are broadcast at 1090 MHz: a decent ADS-B receiver antenna can receive messages from cruising aircraft located up to $400 \mathrm{~km}$ far away, while the range is much lower for aircraft flying in low altitude or on ground.

The collected data used for this study contains a month of ADS-B data collected during October 2019, tagged by the OpenSky Network as flying from or to Zurich airport (LSZH). This airport was chosen for the good coverage offered by the network with about seven receivers in line of sight with 


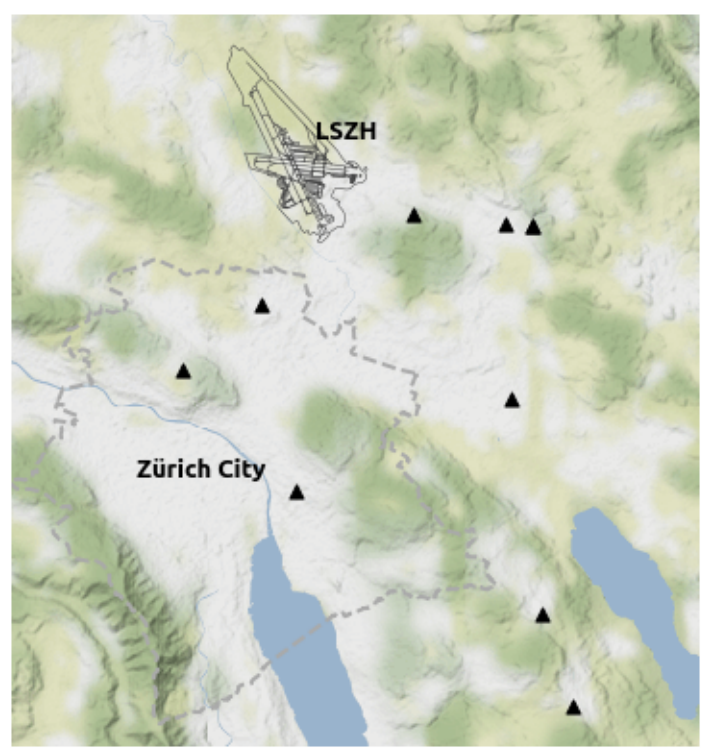

Figure 3. Receivers around Zurich area feeding the OpenSky Network.

the apron (Figure 3) and consistently feeding data of ground trajectories to the network. Recorded data contains timestamps (added on the receiver side, with many receivers equipped with a GPS nanosecond precision clock), transponder unique 24-bit identifiers (icao24), space-filled 8 character callsigns, latitude, longitude (in degrees, 5 digit precision), altitude (in ft, w.r.t. standard atmosphere), ground speeds (in kts), true track angle (in degrees), vertical speed (in kts). Based on sensors located on the landing gear, different messages with similar information are sent when the aircraft is on the ground, resulting in a boolean flag in the OpenSky records.

Aircraft compute their position within uncertainty margins (navigation accuracy) according to actual sensor sources (such as global navigation satellite system, inertial navigation system, or radio navigation) and broadcast their position via ADS-B. The information about the uncertainties are not provided decoded in the OpenSky Network database but could be processed [45] from the raw messages on an as-need basis. However, we kept the uncertainty analysis out of the scope of this paper and chose to manually filter irrelevant data (see Section III-B) as part of the pre-processing step.

As a matter of fact, there are a limitations inherent to the quality of data received:

- reception of trajectories on ground is only possible when active receiving antennas are in line of sight with all taxiing aircraft. This can be made possible with antennas conveniently installed at airports;

- positions of aircraft are computed with embedded inertial systems if satellites are out of sight. This can lead to trajectories not matching the apron structure until the GPS signal is properly caught. Cross-validation with other sources of information (ground radars, signals from other antennas processed for multilateration) is a way to mitigate this issue.

\section{B. Preprocessing of data}

We use the declarative pre-processing grammar of the Python traffic [46] library to describe the pre-processing steps applied to our dataset of trajectories. For instance, the following pre-processing was used for all landing trajectories:

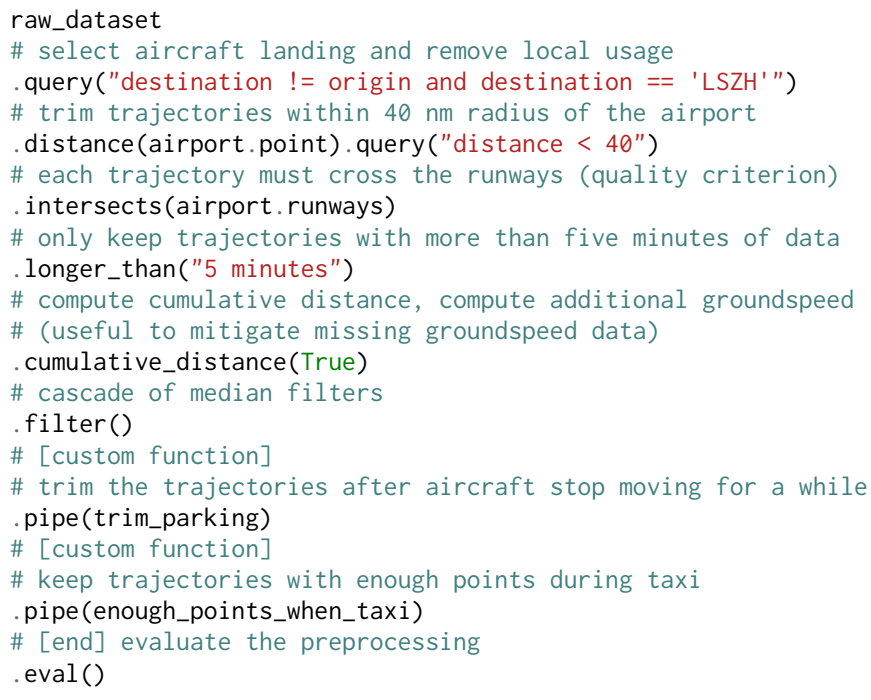

Trajectories collected in the original dataset require specific pre-processing to address several commonly known issues stated above in order to select only trajectories with enough quality in terms of accuracy (the tracks should fit the apron) and precision (a trajectory should consist of enough samples during taxi). Invalid trajectories in our original dataset include:

- ground trajectories computed only from the inertial systems before catching GPS signal (Fig. 4.a)).

- ground trajectories with not enough samples to fully represent the aircraft position at all times (Fig. 4.b)). a) No GPS signal

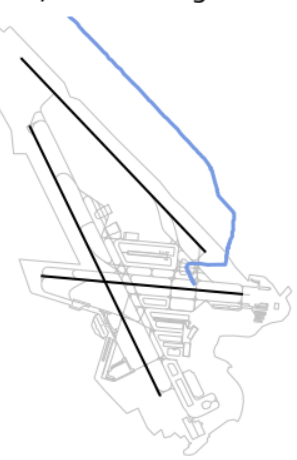

b) Missing points

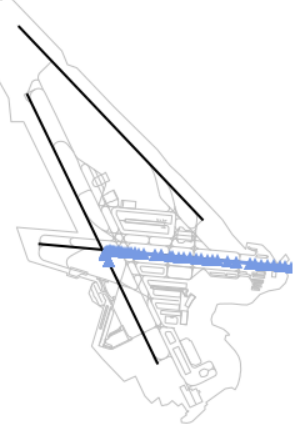

Figure 4. Invalid trajectories detected in the dataset addressing the loss of GPS signal (position changes derived from measures of aircraft acceleration) and missing data points.

\section{Data quality assessment}

The quality of the data has been assessed and compared with data present in Eurocontrol web services, with a preops access. Data available include a flight identifier, callsign, icao24, origin airport, destination airport, estimated off-block time, 
take-off time, and landing time. The aircraft taxi time is also available at the Eurocontrol web services (in theory) but the recorded information are mostly irrelevant, since these data are average values.

The preops access has limitations in data, so cannot be considered as ground truth. Not only do stakeholders not update their information on a regular basis and with significant precision, but also flight plans which are filed manually (about $10 \%$ of all flights) are not available. Over the recorded span from October $5^{\text {th }}$ to $31^{\text {st }}$, 6,315 flights have been seen both on ADS-B and Eurocontrol records, 2,295 flights were only seen on Eurocontrol records and were not sending any ADS-B information, also local receivers recorded Mode $\mathrm{S}$ data from these aircraft, which include callsign, squawk code and altitude, but no positional information. Conversely, 1,299 flights had ADS-B records but no track available on Eurocontrol records (manually filed flight plans).

Figure 5 plots the difference in seconds between the recorded landing time (rounded to the closest minute) in Eurocontrol database and the estimation of our third milestone ALDT (actual landing time). Considering a precision of 60 seconds in the Eurocontrol database, the distribution of errors validates the estimation of this particular milestone using both aircraft emitted ADS-B messages and our A-CDM lite approach.

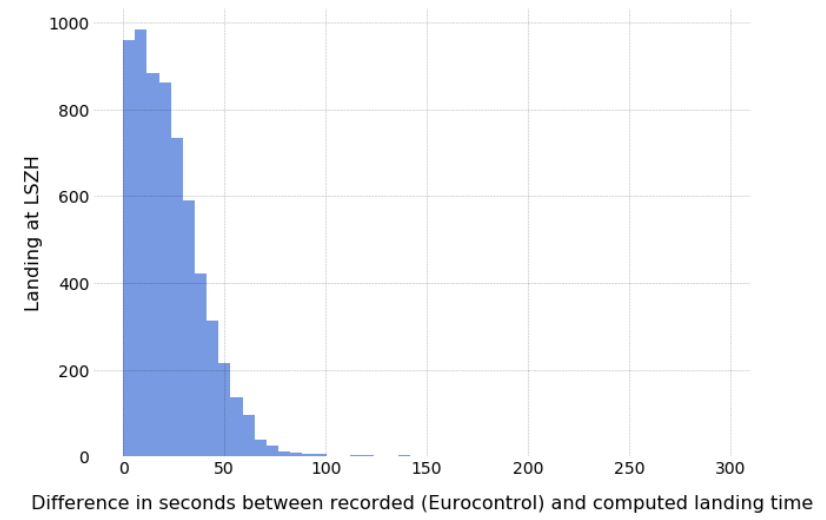

Figure 5. Difference (absolute), in seconds, between (automatically) recorded landing time in Eurocontrol database and our ALDT (actual landing time) milestone.

Figure 6 plots the distribution of taxi times, rather short on average in Zurich airport. A more detailed distribution is analysed in depth in Section IV. The distribution exhibited on Figure 6 is more striking for the 1500+ flights with positional data no longer recorded after the runway exit. This is possibly related with a receiver having this part of the airport apron in line of sight being offline at these moments. For the best mitigation measure the authors can think of, is to convince airports of the benefits of our data-driven A-CDM lite approach and convince them to have (multiple) receivers on-site feeding the network.

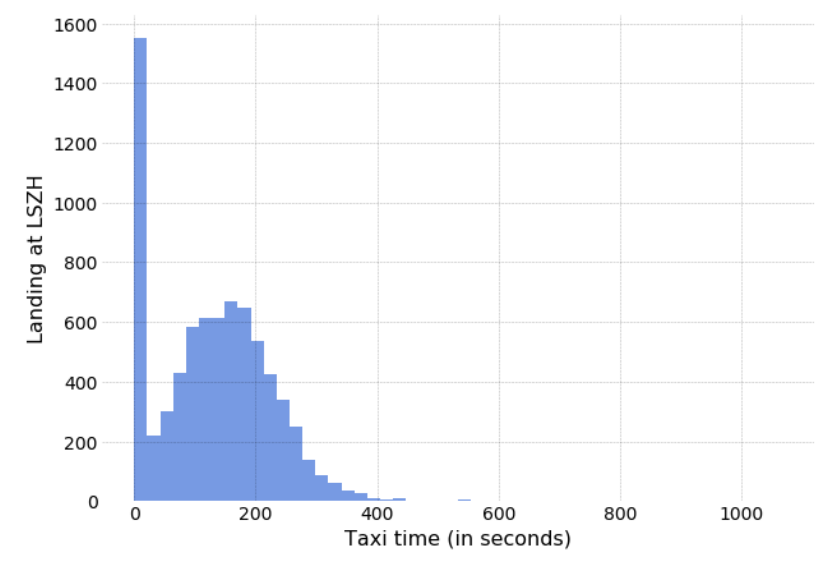

Figure 6. Distribution of aircraft taxi times in seconds, from runway exit to last recorded position.

\section{Data compression - simplification}

Aircraft trajectories are a list of status changes, which are often contains a linear path elements, interrupted by changes in both altitude and direction. Thus, the recorded data contains several data points with no additional information, which could be deleted. This compression process results in a data reduction of approx. $80 \%$ [29]. If at later stage a higher resolution of the trajectory could be create a linear resampling. Nevertheless, the compression has to assure that particular points of interests will be part of the compressed (simplified) trajectory. The application of the Ramer-Douglas-Peucker (RDP) algorithm [47] shows an appropriate behaviour, when the aircraft trajectory is divided into a ground and an aerial part. For both parts different values for precision (maximum distance between simplified and original trajectory) are taken to decide if a status update will add new information (add to the simplified list) or could be described by linear combination of two other status changes (not to be considered further). As Figure 7 exhibits, setting the distance to $100 \mathrm{~m}$ and $25 \mathrm{~m}$ reduces the initial number of positions from 67 to 7 and 13, respectively.
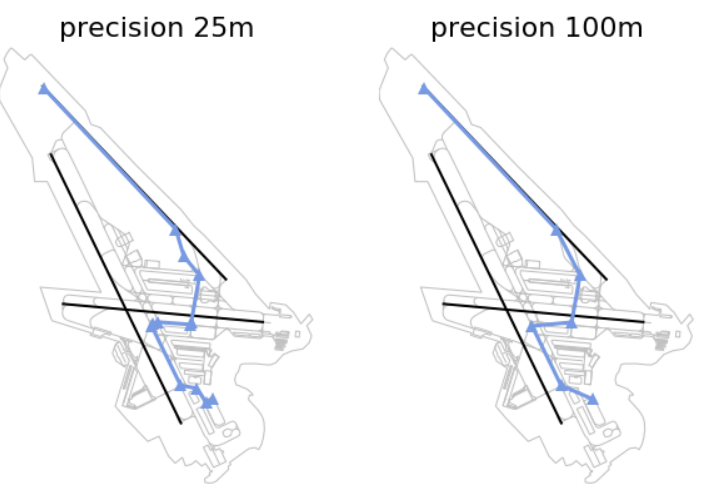

Figure 7. Compression of exemplary ground trajectory of arriving aircraft at Zurich airport landing on runway 14: operational milestones and maximum distance between origin and simplified trajectory using a maximum distance (precision) of $25 \mathrm{~m}$ (left) and $100 \mathrm{~m}$ (right). 
Since the average position error is about $21 \mathrm{~m}$, identified during radar verification [48], a distance of $25 \mathrm{~m}$ was finally implemented for ground operations. In particular, ground operations exhibits the need of an additional parameter to be included in the simplification/compression process: aircraft speed. For that reason, Figure 8 (top) exhibits the deficiencies in reproducing aircraft speed, when only considering the aircraft location. An additional consideration of the aircraft speed changes with a threshold of $4 \mathrm{~m} \cdot \mathrm{s}^{-1}$ increases the number of trajectory positions to 23 , see Figure 8 (below). Finally, we propose a trajectory compression with the following setting for location (latitude, longitude), altitude and speed profiles for the RDP algorithm on the ground (in the air): $25 \mathrm{~m}$ (resp. $200 \mathrm{~m}), 0 \mathrm{~m}$ (resp. $30 \mathrm{~m}), 4 \mathrm{~m} \cdot \mathrm{s}^{-1}\left(\right.$ resp. $\left.10 \mathrm{~m} \cdot \mathrm{s}^{-1}\right)$.

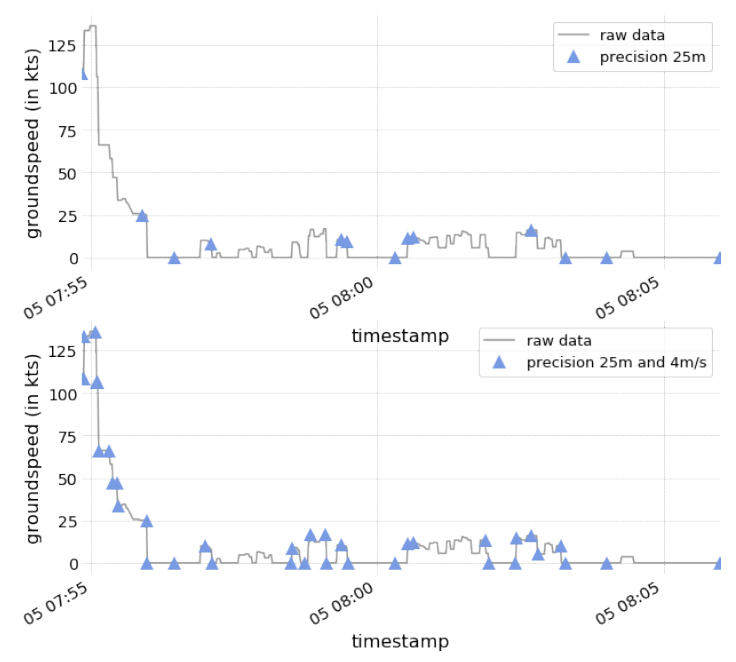

Figure 8. Speed changes during ground operations are not sufficiently covered by using location-based simplification algorithms only.

As Figure 7 and 8 show, the RDP algorithm works as an edge/corner detector, identifying deflection points at the apron. This behavior identifies operational hotspots [30], by means of areas, which are of particular interests to apron controllers. Often detected hotspots in a similar timeframe are precursors for bottlenecks (like waiting queues at runway access) and could be used as a starting point for operational optimizations. Furthermore, these corners could also be used to identify operational milestones, such as landing or take-off time.

\section{ANALYSIS OF AIRPORT OPERATIONAL ENVIRONMENT}

In this section, we show four examples of application of the derived milestone concept: (A) aircraft inbound flow into Zurich airport, (B) clustering of ground trajectories, and determination of (C) runway occupancy and (D) taxi times. These examples emphasize the most relevant parameters, which heavily affect airport performance. Inbound trajectories determine landing times and are part of the airport arrival management to ensure an efficient use of runway capacity. After the aircraft landed, the runway occupancy time determine the further use of this particular runway, and dependent operations at other runways, for arrival and departure procedures [28].
After the aircraft leaves the runway and enters the taxiway system, interactions with all active aircraft and ground vehicles determine the (variable) taxi time (VTT) of the actual aircraft as well as the times of all other affected aircraft (Fig. 1, Sec. II). Beside the common concept of data sharing and the introduced milestone approach, A-CDM also addresses the determination of variable taxi times. Instead of using default pre-defined values for aircraft taxiing as reflected in operational databases, the calculation of VTT and finally the provision of taxi time forecasts will enable an optimize use of the apron/taxi capacities.

\section{A. Flows into airport}

The specific ASMA transit time depends on the direction of the incoming traffic flow. Therefore, Eurocontrol suggest to divide this circular area around the airport (radius of 40NM) into angular sectors by entry bearings [44], depending on the distribution of the inbound traffic. As Figure 9 exhibits, the consideration of bearings as single input will not reflect the nature of arrival procedures. Thus, arrival management systems allow a continuous optimisation of arrival traffic sequences using appropriate tactical intervention by air traffic controllers to maximise the airport capacity.

Trajectory clustering in terminal manoeuvring areas [18], [49] is a powerful tool to analyse the inbound traffic to an airport. Figure 9 displays few trajectories from four different clusters of aircraft coming from the North of the airfield.
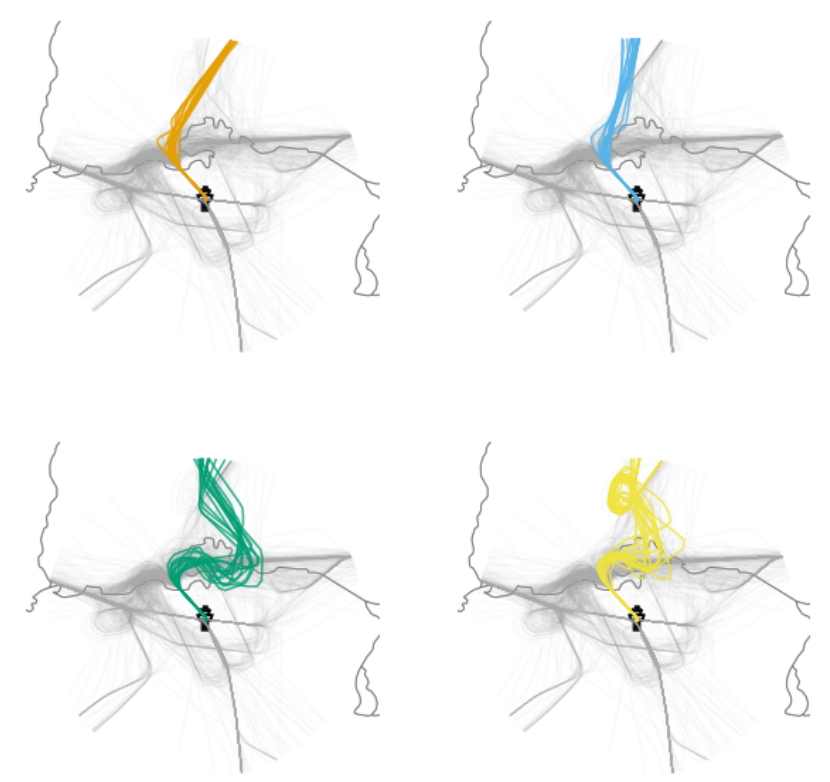

Figure 9. Clustering among northbound arrival flows at Zurich airport.

Clustering yields different flows according to the amount of scheduling work: the first cluster gathers trajectories flying directly to catch the signal of the instrument landing system (ILS) on runway 14 . The second cluster shows a very slight level of sequencing, the third cluster reflects a low level of disruption with tromboning into final approach while the last 
one shows a higher level of disruption, with an ASMA transit time 2.5 times longer than in smooth configuration.

Given characteristic demand/flow constellations, runway operational concepts, and weather conditions, specific arrival procedures will be applied by air traffic controllers. Thus, the determination of both unimpeded and additional ASMA transit times have to consider the operational situation at the airport and an optimal queuing of aircraft needed to enable maximum utilization of airport capacity. Analyses about airport congestion and traffic in Terminal Manoeuvring Areas (TMA) show a big discrepancy in specific ASMA transit times, namely the actual time spent by a flight between its last entry in the cylinder (entry-time at $40 \mathrm{NM}$ upstream) and the actual landing time (Figure 10). An efficient prediction of the configuration and disruption mode at the airport can improve the prediction of ASMA transit times according to the violin plots on Figure 10.

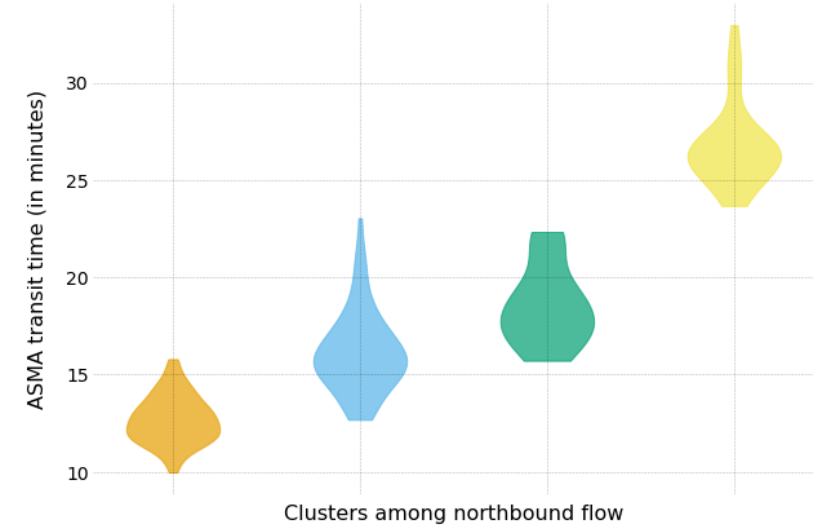

Figure 10. Analysis of ASMA transit times before landing.

\section{B. Runway occupancy time}

Figure 11 shows an exemplary analysis of the runway occupancy time of runway 14 (northern runway, landing in southerly direction), indicating the use of different runway exits. After landing, aircraft should leave the runway in a fast way, since following aircraft are only allowed to use a cleared runway. In times of high intense runway operations or to cover uncertainties in the actual landing time (ALDT), safety will be assured by introducing spacing buffers to prevent separation infringements. The size of these buffers affect the airport capacity and mainly depends on individual controller experiences, mix of aircraft types (wake turbulence category [50]), type of operations (arrival, departure), and weather conditions [38].

\section{Ground trajectories}

Clustering of ground trajectories is a challenging task because of the difficulty to find a proper distance function between them. Moreover, after resampling trajectories to enough samples to grasp the structure of the traffic, vectors of very high dimensions will be passed to the clustering algorithm and the curse of dimensionality will hit. A common

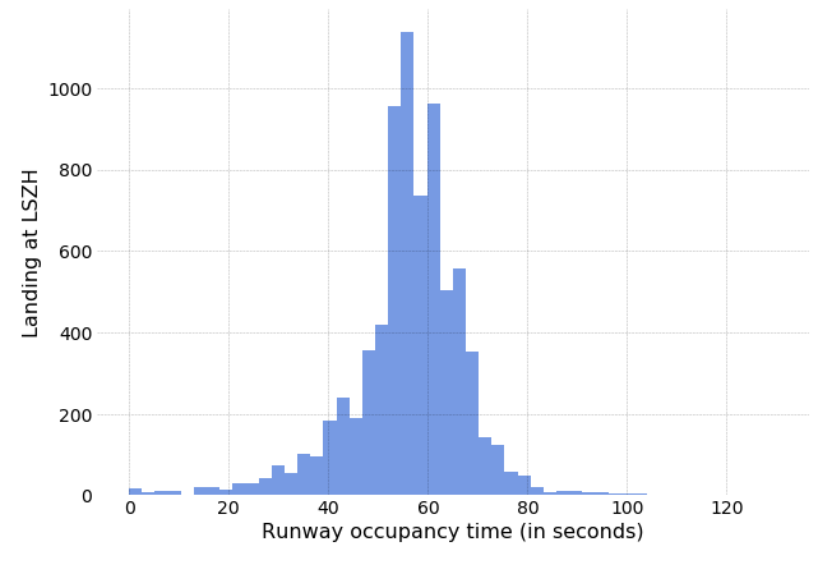

Figure 11. Distribution of runway occupancy times at runway 14 (upper north runway), measured as time between aircraft landing time and time when the aircraft is leaving the runway.

workaround is to project highly dimensional samples into a lower dimensional space and to perform clustering in this new space. A wide variety of techniques are available to compute relevant projections into a lower dimensional space, like Principal Component Analysis (PCA), autoencoders or tSNE [51]. Fig. 12 plots a small subset of the clusters resulting from a DBSCAN [52] clustering on the two dimensional space where t-SNE projected the trajectories, consisting of 50 samples of latitudes, longitudes and ground speeds. The clusters reflect various typical ground trajectories followed by aircraft after landing.
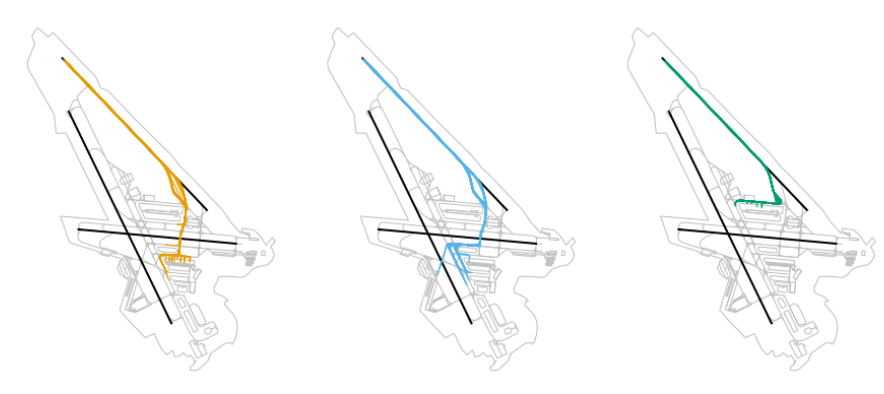

Figure 12. A subset of the clustered aircraft ground trajectories detected using positional features provided by ADS-B data.

\section{Taxi times}

Taxi time is a major contributor to airport performance. The whole taxi system needs to be efficiently managed to drive aircraft seamlessly to and from the runway. Zurich airport consists of a set of dependent and crossing runways, which is normally accompanied with corresponding waiting areas to allow safe runway crossings (see Fig. 13).

According to the airport and runway layout, it is expected that different average taxi times and deviations exist. As Fig. 14 exhibits, the operating runway significantly impacts the aircraft taxi time. In this context, runway 28 (red line) is mostly used in evening hours (from 21:30 h during weekdays) 


\section{DISCUSSION AND CONCLUSION}

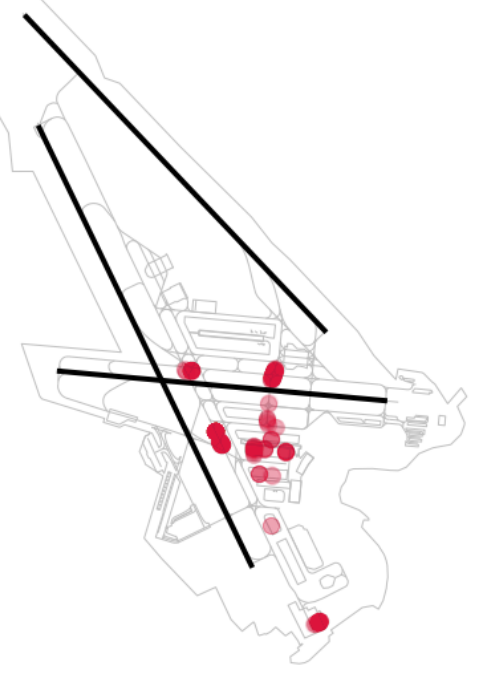

Figure 13. Density map of aircraft positions at Zurich airport with aircraft ground speed $=0$.

when the traffic demand (and flow) is significantly lower than over the day. If runway 14 is used for aircraft arrival, the taxi times indicate a bi-modal distribution (blue line).

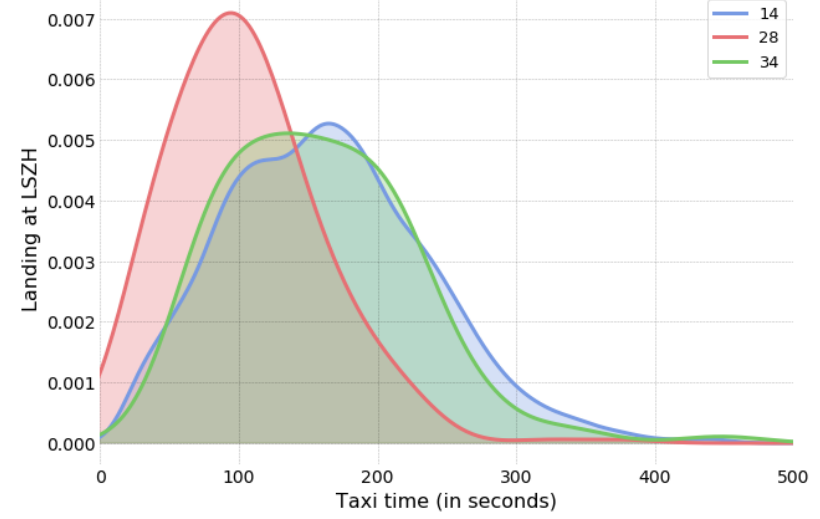

Figure 14. Distribution of taxi times for trajectories with enough points during taxi, and splitted according to the runway in use (14 - landing from north (left runway), 28 - landing from east, or 34 - landing from south (left runway)).

For the calculation of variable taxi times (VTT), the positions of the ground trajectories could be further processed and clustered. As mentioned, the applied RDP algorithm detect non-linear changes in aircraft location and speed, which are also associated with intersections within the runway/taxi system. These positions are clustered with a Kernel Density Estimation approach (DENCLUE algorithm [53]), where the positions are assigned to clusters by hill climbing. This clustering process results in a representation of operational hot spots [30], which will be the basis for a graph representation of the apron network. Finally, this graph will be used for VTT prediction in our follow up research.
The analysis of available ADS-B data at Zurich airport, which currently offers a decent coverage on ground shows interesting patterns in taxiing and correlations found with traffic density that, are consistent with what we would expect from the way airports are usually operated. This proof of concept for our tailored milestones concept shows promising results with regards to the analysis of the operational performance at the airport. Thus, we are confident that an implementation of A-CDM lite will provide an appropriate performance-based airport management, when ADS-B becomes mandatory in 2020. The use of ADS-B is convenient as it is very costeffective: the installation of a decent decoding installation nearby airports costs less than $1,000 €$ (with most basic receivers, costs going down to $20 €$ ), making replication and validation of positions by multilateration an option to address spoofing attacks.

This study only analyses the airside trajectory part with open sources for aircraft movement data, which validates part of the concept. Future works will include the incorporation of flight plan and operational history data accessible through Eurocontrol B2B services, for a further validation of the concept and for improving the predictive power of our approach. The inclusion of more sources of data to address possible causes of disruptions (such as weather [31], [32], [38]) and specific operational behaviours at the airport will also be considered.

We introduced a tailored approach of the A-CDM milestone concept (A-CDM lite), which contains of ten operational milestones along the aircraft trajectory. These milestones can be fully derived from public available ADS-B messages emitted by arriving and departing aircraft. In particular, taking the first received message from a departing aircraft (crew activates the ADS-B transponder) as 'aircraft ready' milestone, seems to be a promising approach to predict the aircraft off block time. Our contribution addresses four important elements of the airport management: arrival flows, runway occupancy, ground trajectories and taxi times. We provide first analysis of the airport operational performance using data analytics techniques and machine learning approaches.

The benefits of A-CDM lite implementation are threefold: (1) awareness of local, actual airport operations, (2) knowledge about the performance of connected airports (analysing their local operations), and (3) provide milestone data from A-CDM lite to the network manager to improve the performance of air traffic system. A full implementation of A-CDM is costintensive and not preferred by small/medium airports.

Our A-CDM lite concept shows that the use of ADS-B data allows to derive a set of milestones to manage an airport with performance targets. We expect that our A-CDM lite will convince airport operators to implement the reduced milestone concept and significantly boost the availability of local data for an improved management of the whole traffic system. Our next research will include the stepwise prediction of operational milestones to provide target times for operations and the test implementation within an operative airport environment. 


\section{ACKNOWLEDGEMENTS}

This work is partially supported by NTU-CAAS Research Grant M4062429.052 by Air Traffic Management Research Institute, School of MAE, NTU, Singapore.

\section{REFERENCES}

[1] Eurocontrol Airport CDM Team, "Airport CDM implementation manual, ver. 5," 2017.

[2] S. Pickup, "A-CDM impact assessment," 2016.

[3] SESAR JU, "European ATM Master Plan," 2015.

[4] F. Piekert, A. Marsden, and O. Delain, "Europes next step in Airport Performance Management Research.” in EIWAC, 2017.

[5] Airports Council International Europe, "SESAR and the Digital Transformation of Europe's airports," 2018

[6] S. Corrigan, L. Martensson, A. Kay, S. Okwir, P. Ulfvengren, and N. McDonald, "Preparing for Airport Collaborative Decision Making (A-CDM) implementation: an evaluation and recommendations." in Cognition Technology and Work, 2015.

[7] European Telecommunications Standards Institute, "ETSI EN 303212 V1.1.1," 2010

[8] Eurocontrol, "Airport CDM Cost Benefit Analysis," 2008.

[9] EU, "Commission impl. regulation (EU) No 1207/2011," 2011.

[10] — "Commission impl. regulation (EU) No 2017/386," 2017.

[11] FAA, "14 CFR, FAR section 91.225 Automatic Dependent SurveillanceBroadcast (ADS-B) Out equipment and use"," 2015.

[12] M. Schäfer, M. Strohmeier, V. Lenders, I. Martinovic, and M. Wilhelm, "Bringing up OpenSky: A large-scale ADS-B sensor network for research," in Proceedings of the 13th international symposium on Information processing in sensor networks, 2014, pp. 83-94.

[13] T. Delovski, K. Werner, T. Rawlik, J. Behrens, J. Bredemeyer, and R. Wendel, "ADS-B over satellite the worlds first ADS-B receiver in space," in mall Satellites Systems and Services Symposium, 2014.

[14] SESAR Deployment Manager, "ADS-B and other means of surveillance implementation status," 2018.

[15] L. Basora, V. Courchelle, J. Bedouet, and T. Dubot, "Occupancy peak estimation from sector geometry and traffic flow data," in 8th SESAR Innovation Days, 2018.

[16] M. Gariel, A. N. Srivastava, and E. Feron, "Trajectory clustering and an application to airspace monitoring," IEEE Transactions on Intelligent Transportation Systems, vol. 12, no. 4, pp. 1511-1524, 2011.

[17] L. Basora, J. Morio, and C. Mailhot, "A trajectory clustering framework to analyse air traffic flows," in 7th SESAR Innovation Days, 2017.

[18] X. Olive and J. Morio, "Trajectory clustering of air traffic flows around airports," Aerospace Science and Technology, vol. 84, pp. 776-781, 2019.

[19] X. Olive, J. Grignard, T. Dubot, and J. Saint-Lot, "Detecting controllers actions in past Mode S data by autoencoder-based anomaly detection," in 8th SESAR Innovation Days, 2018.

[20] S. Das, B. L. Matthews, A. N. Srivastava, and N. C. Oza, "Multiple kernel learning for heterogeneous anomaly detection: algorithm and aviation safety case study," in Proc. of the 16th international conference on Knowledge discovery and data mining, 2010, pp. 47-56.

[21] X. Olive and P. Bieber, "Quantitative assessments of runway excursion precursors using Mode S data," in Proc. of the International Conference for Research in Air Transportation, 2018.

[22] L. Basora, X. Olive, and T. Dubot, "Recent Advances in Anomaly Detection Methods Applied to Aviation," Aerospace, vol. 6, no. 11, p. 117, 2019.

[23] Y. Lv, Y. Duan, W. Kang, Z. Li, and F. Wang, "Traffic flow prediction with big data: A deep learning approach." IEEE Transactions on Intelligent Transportation Systems, vol. 16, no. 2, pp. 865-873, 2015.

[24] C. Di Ciccio, H. Van der Aa, C. Cabanillas, J. Mendling, and J. Prescher, "Detecting flight trajectory anomalies and predicting diversions in freight transportation," Decision Support Systems, vol. 88, pp. 1-17, 2016.

[25] Y. Liu, M. Hansen, D. J. Lovell, and M. O. Ball, "Predicting aircraft trajectory choice - a nominal route approach," in Proc. of the International Conference for Research in Air Transportation, 2018.

[26] I. Gerdes, A. Temme, and M. Schultz, "Dynamic airspace sectorization using controller task load," in 6th SESAR Innovation Days, 2016.

[27] _ - "Dynamic airspace sectorisation for flight-centric operations," Transportation Research Part C: Emerging Technologies, vol. 95, pp. 460-480, 2018.
[28] F. Herrema, R. Curran, S. Hartjes, M. Ellejmi, S. Bancroft, and M. Schultz, "A machine learning model to predict runway exit at vienna airport," Transportation Research Part E: Logistics and Transportation Review, vol. 131, pp. 329-342, 2019.

[29] M. Schultz, J. Post, and S. Alam, "Concept of a data-driven airport management using ADS-B data," in EIWAC, 2019.

[30] M. Schultz, J. Rosenow, and X. Olive, "A-CDM lite: situation awareness and decision making for small airports based on ADS-B data," in 9th SESAR Innovation Days, 2019.

[31] S. Reitmann and M. Schultz, "Computation of air traffic flow management performance with long short-term memories considering weather impact," Lecture Notes in Computer Science, vol. 11140, 2018.

[32] S. Reitmann, S. Alam, and M. Schultz, "Advanced quantification of weather impact on air traffic management - intelligent weather categorization with machine learning." in 13th USA/Europe ATM R\&D Seminar, 2017

[33] M. Schultz and S. Reitmann, "Machine learning approach to predict aircraft boarding," Transportation Research Part C: Emerging Technologies, vol. 98, pp. 391-408, 2019.

[34] J. Sun, J. Ellerbroek, and J. M. Hoekstra "Aircraft initial mass estimation using Bayesian inference method," Transportation Research Part C: Emerging Technologies, vol. 90, 2018.

[35] J. Sun, "Open Aircraft Performance Modeling Based on an Analysis of Aircraft Surveillance Data," Ph.D. dissertation, Delft University of Technology, 2019.

[36] S. Helm, S. Loth, and M. Schultz, "Advancing Total Airport Management." in ATRS, 2015.

[37] Y. Günther, A. Pick, S. Kern, S. Lorenz, M. Schultz, T. Gerz, F. Keis, and M. Köhler, "Efficient airport management under severe weather conditions," in International Symposium on Enhanced Solutions for Aircraft and Vehicle Surveillance Applications, 2016.

[38] M. Schultz, S. Lorenz, R. Schmitz, and L. Delgado, "Weather impact on airport performance," Aerospace, vol. 5(4), 2018.

[39] H. Fricke and M. Schultz, "Delay impacts onto turnaround performance," in 9th USA/Europe ATM R\&D Seminar, 2009.

[40] B. Oreschko, M. Schultz, J. Elflein, and H. Fricke, "Significant turnaround process variations due to airport characteristics," Air Transport and Operations, pp. 263-270, 2010.

[41] M. Schultz, "Fast aircraft turnaround enabled by reliable passenger boarding," Aerospace, vol. 5, no. 1, p. 8, 2018

[42] _ - "A metric for the real-time evaluation of the aircraft boarding progress," Transportation Research Part C: Emerging Technologies, vol. 86, pp. 467-487, 2018.

[43] Harris Orthogon GmbH, "OSYRIS Pre-Departure Sequencer," 2015.

[44] L. Cappelleras, "Additional Asma Time Performance Indicator Document, 00-06 Eurocontrol/PRU," 2015.

[45] J. Sun, H. Vû, J. Ellerbroek, and J. M. Hoekstra, "pymodes: Decoding mode-s surveillance data for open air transportation research," IEEE Transactions on Intelligent Transportation Systems, 2019.

[46] X. Olive, "traffic, a toolbox for processing and analysing air traffic data," Journal of Open Source Software, vol. 4, no. 39, p. 1518, 2019.

[47] D. H. Douglas and T. K. Peucker, "Algorithms for the reduction of the number of points required to represent a digitized line or its caricature," Cartographica: The International Journal for Geographic Information and Geovisualization, vol. 10, no. 2, pp. 112-122, 1973.

[48] T. Verbraak, J. Ellerbroek, J. Sun, and J. Hoekstra, "Large-scale ADSB data and signal quality analysis." in 12th USA/Europe ATM $R \& D$ Seminar, 2017.

[49] X. Olive and L. Basora, "Air Traffic Data Processing using Python: Trajectory Clustering," in Proc. of the 7th OpenSky Workshop, 2019.

[50] ICAO, "Procedures for Air Navigation Services Air Traffic Management (PANS-ATM, Doc 4444)," 2016.

[51] L. van der Maaten and G. Hinton, "Visualizing High-Dimensional Data using t-SNE," Journal of Machine Learning Research, vol. 9, 2008.

[52] M. Ester, H.-P. Kriegel, J. Sander, X. Xu et al., "A density-based algorithm for discovering clusters in large spatial databases with noise." in Proc. of the 2nd International Conference on Knowledge Discovery and Data Mining, vol. 96, no. 34, 1996.

[53] A. Hinneburg and H.-H. Gabriel, "DENCLUE 2.0: Fast clustering based on Kernel Density Estimation," in Advances in Intelligent Data Analysis VII, 2007 